\title{
Investigation of irradiation conditions for recurrent breast cancer in JRR-4
}

\author{
H. Horiguchi ${ }^{1}$, T. Nakamura ${ }^{1}$, H. Kumada ${ }^{2}$, H. Yanagie ${ }^{3,4}$, M. Suzuki ${ }^{5}$ and H. Sagawa ${ }^{1}$ \\ ${ }^{1}$ Department of Research Reactor and Tandem Accelerator, Japan Atomic Energy Agency, \\ Tokai, Ibaraki, Japan \\ ${ }^{2}$ Proton Medical Research Centre, University of Tsukuba, Tsukuba, Ibaraki, Japan \\ ${ }^{3}$ Department of Nuclear Engineering and management, Graduate School of Tokyo, Tokyo, Japan \\ ${ }^{4}$ Cooperative Unit of Medicine and Engineering, University of Tokyo Hospital, Tokyo, Japan \\ ${ }^{5}$ Particle Radiation Oncology Research Center, Kyoto University, Osaka, Japan
}

\begin{abstract}
Clinical trials of boron neutron capture therapy (BNCT) for recurrent breast cancers are being considered at Japan Research Reactor No.4 (JRR-4). In this study, the irradiation technique for a total mastectomy patient with recurrent cancer was optimized by dosimetric calculations using JAEA computational dosimetry system (JCDS). The evaluation was performed using an en face technique and a tangents technique with thermal neutron beam mode at JRR-4. The results revealed that equivalent doses of lung, heart, liver and skin were similar in each irradiation technique due to the isotropic scattering of thermal neutrons in the body. On the other hand, the irradiation time with the tangents technique was a few times longer than with the en face technique. We concluded that the en face technique was an optimal irradiation technique for recurrent breast cancers using thermal neutron beam mode in terms of shorter irradiation time and easier patient positioning.
\end{abstract}

Keywords: boron neutron capture therapy, recurrent breast cancer, treatment planning, JRR-4

\section{Introduction}

Clinical trials for BNCT have been performed at JRR-4 (Nakagawa et al., 2003, Yamamoto et al., 2009). Current BNCT trials at JRR-4 are applied for malignant brain tumor, head and neck cancer, skin cancer. Recurrent breast cancers are being considered as a new application of BNCT trials at JRR-4. Breast cancers might recur on local sites or on distant sites from the breast after primary resection. Other radiation therapies are conducted to treat patients of recurrent breast cancers. If sufficient boron compounds can be accumulated to the tumor, BNCT trials can be applied to these patients (Yanagie et al., 2009).

In this study, optimal irradiation techniques for recurrent breast cancers were investigated using the JRR-4 neutron beam facility. A thorax model was created as a patient of total mastectomy breast with cancers by using the computed tomography (CT) images. We studied the preliminary dosimetry for this actual patient model by JCDS which was a treatment planning system developed by JAEA (Kumada et al., 2007). To investigate the effectiveness of different irradiation techniques, the evaluation was performed using en face technique and tangents technique with thermal neutron beam mode at JRR-4. With external photon beam radiation, the tangents technique is generally used for breast and chest wall treatment to reduce healthy organ doses.

\section{Materials and Methods}

\subsection{Calculation model}

The attempt for optimizing the irradiation technique for patients with recurrent breast cancer was performed using an actual thorax model by the treatment planning system JCDS. The model of the 
recurrent breast cancer patient was created by using the CT images. The model consisted of the soft tissues, the bone, the skin, the lungs, the heart and the liver shown in Figure 1. The recurrent tumor was a simulated tumor located in the left side of the thorax surface after surgical resection treated as total mastectomy breast. The tumor was about $100 \mathrm{~mm}$ in diameter and thickness was $5 \mathrm{~mm}$. The regions of interest (ROI) were placed on the tumor, the skin, the right lung, the left lung, the heart and the liver in this model. The ROI of the skin was located on the body surface including the tumor surface area. The materials were defined according to ICRU report 46 (1992).

\subsection{Overview of JCDS}

We applied JCDS to perform the neutron and photon dosimetry in this study. JCDS is a dose planning system which is developed to carry out the BNCT trials based on accurate dosimetry (Kumada et al., 2007). To simulate the irradiations, JCDS constructs a three-dimensional model of the thorax based on CT images. By using CT images, organs in the thorax model such as the soft tissues and the bone are automatically differentiated based on CT values. JCDS creates a voxel model of the thorax consisting of $5 \times 5 \times 7 \mathrm{~mm}^{3}$ voxel cells to perform the particles transport calculation with MCNP efficiently. MCNP is a general purpose Monte-Carlo n-particle transport code (Briesmeister, 2000).

\subsection{Assumption for treatment planning}

The concentration of ${ }^{10} \mathrm{~B}$ in the blood was assumed $24 \mu \mathrm{g} / \mathrm{g}$. Tumor-blood ratio and skin-blood ration of boron concentration was 3.0 and 1.2, respectively. These parameters were approximately same as our current treatment protocol for head and neck cancers which uses para-boronophenylalanine (BPA). The other parameters in this study were summarized in Table 1 . The compound biologic effectiveness (CBE) factors of skin, lung and liver were assumed 2.5, 2.3 and 4.25, respectively (Fukuda et al., 1994, Kiger et al., 2008, Suzuki et al., 2000). The relative biologic effectiveness (RBE) factors of ${ }^{1} \mathrm{H}(\mathrm{n}, \mathrm{n}$ ')p reaction (fast neutron dose) and ${ }^{14} \mathrm{~N}(\mathrm{n}, \mathrm{p}){ }^{14} \mathrm{C}$ reaction (nitrogen dose) were assumed to be the value of 2.5. The RBE factor of gamma ray dose was assumed to be 1.0. The CBE factors and the RBE factors were used to convert physical dose (Gy) to photon equivalent dose (Gy-Eq).

To optimize the irradiation technique for recurrent breast cancer, we evaluated the effectiveness as reducing the neighbor healthy organs doses with a single field technique and a multiple field technique. The multiple field technique, which was performed by a double-fields irradiation of tangents technique, was compared with a single-field irradiation of en face technique. Figure 2 shows the irradiation position of MCNP calculation. The en face technique was planned to homogeneously deliver the neutron beam to whole tumor region. The tangents technique was planned from lateral and medial tangent field with oblique angle of 60 degrees to the en face position. To compare the dose distributions and irradiation time between each irradiation technique, these treatment plans were normalized by the minimum equivalent dose of tumor at $30 \mathrm{~Gy}$-Eq. We performed the dosimetry using these assumptions with thermal neutron beams of JRR-4 (JRR4-TNB2). JRR4-TNB2 was an optimal beam mode for a tumor spread on the breast surface because of low epithermal and fast neutrons contamination at beam aperture (Torii et al., 2000).

\section{Results and discussion}

Table 2 summarizes the equivalent doses for the tumor and healthy organs by the en face technique and the tangents technique. To compare the equivalent dose of the tumor and healthy organs in each irradiation technique, these treatment plans were normalized by the minimum equivalent dose of tumor at $30 \mathrm{~Gy}$-Eq. The single-field irradiation delivered $65.0 \mathrm{~Gy}$-Eq as a maximum tumor dose, 18.2 Gy-Eq as maximum skin dose, 9.8 Gy-Eq as a maximum left lung dose, 3.7 Gy-Eq as a maximum heart dose, 


\subsection{Gy-Eq as a maximum liver dose.}

The tangents technique was performed as irradiations from two directions which were merged lateral tangent and medial tangent. The total equivalent dose of the tangents technique delivered 66.8 Gy-Eq as a maximum tumor dose, $19.5 \mathrm{~Gy}$-Eq as a maximum skin dose, $8.3 \mathrm{~Gy}$-Eq as a maximum left lung dose, 3.4 Gy-Eq as a maximum heart dose and 2.1 Gy-Eq as a maximum liver dose. The tangents technique provided comparable results on the tumor dose to the en face technique and delivered a slightly reduced maximum dose on the lung and the heart. On the other hand, the tangents technique delivered slightly higher skin dose than the en face technique. The mean doses and minimum doses of healthy organs (lung, heart, liver) were almost same doses in each irradiation technique. The irradiation time was $40.2 \mathrm{~min}$ and $118.7 \mathrm{~min}$ by the en face technique and by the tangents technique, respectively. The tangents technique was not suitable as our treatment technique for recurrent breast cancer due to the long irradiation time $(\sim 2 \mathrm{~h})$.

Figure 3 shows equivalent dose distributions calculated for lung tissue with the en face technique (Figure 3a) and with the tangents technique (Figure 3b) on the thorax cross section. The isodose distribution with the tangents technique was merged the lateral tangent (Figure 3c) and the medial tangent (Figure 3d) by using merger option of JCDS. The isodose distribution of the tangents technique was similar to the dose distribution of the en face technique, particularly in deeper region of the body. The results revealed that the equivalent dose of the lung was similar in each irradiation technique due to the isotropic scattering of thermal neutrons in the body.

Our assumption of boron concentration in the tumor has not been elucidated yet. It is important for accurate dosimetry to understand the boron concentration of the tumor by pharmacokinetics method such as ${ }^{18} \mathrm{~F}$-BPA-PET technology. If sufficient boron compound can be accumulated to breast tumors, the BNCT trials can be applied to the recurrent breast cancers. Furthermore, to evaluate accurate dosimetry for patients suffering from recurrent breast cancer, we will investigate the respiratory motion of patient and introduce this motion effect to our preliminary dosimetry.

\section{Conclusions}

This study showed the optimal irradiation technique for BNCT dosimetry of recurrent breast cancers. The en face technique with thermal neutron beam was optimal technique due to shorter irradiation time and easier positioning of patient. The tangents technique was an advantage of maximum tumor, lung and heart dose over the en face technique. But, longer irradiation time was not suitable for actual clinical trials. We are performing further investigations with different patient models and techniques for recurrent breast cancers.

\section{References}

Briesmeister, J.F. (Ed.), 2000. MCNP-A General Monte Carlo Code N-Particle Transport Code Version 4C, LA-13709-M

Fukuda, H., Hiratsuka, J., Honda, C., Kobayashi, T., Yoshino, K., Karashima, H., Takahashi, J., Abe, Y., Kanda, K., Ichihashi, M., Mishima, Y., 1994. Boron neutron capture therapy of malignant melanoma using 10B-paraboronophenylalanine with special reference to evaluation of radiation dose and damage to the normal skin. Radiat. Res. 138 (3), 435-442.

International Commission on Radiation Units and Measurements, 1992. Photon, electron, proton and neutron interaction data for body tissues. ICRU Report 46.

Kiger, J.L., Kiger, W.S.3rd, Riley, K.J., Binns, P.J., Patel, H., Hopewell, J.W., Harling, O.K., Busse, P.M., Coderre, J.A., 2008. Functional and histological changes in rat lung after boron neutron capture therapy. Radiat. Res. 170 (1), 60-69. 
Kumada, H., Yamamoto, K., Matsumura, A., Yamamoto, T., Nakagawa, Y., 2007. Development of JCDS, a computational dosimetry system at JAEA for boron neutron capture therapy. J. Phys.: Conf. 74, 021010.

Nakagawa, Y., Pooh, K., Kobayashi, T., Kageji, T., Uyama, S., Matsumura, A., Kumada, H., 2003. Clinical review of the Japanese experience with boron neutron capture therapy and a proposed strategy using epithermal neutron beams. J. Neuro-Oncol. 62 (1), 87-99.

Suzuki, M., Masunaga, S., Kinashi, Y., Takagaki, M., Sakurai, Y., Kobayashi, T., Ono, K., 2000. The effects of boron neutron capture therapy on liver tumors and normal hepatocytes in mice. Jpn. J. Cancer Res. 91 (10), 1058-1064.

Torii, Y., Yamamoto, K., Kishi, T., Hori, N., Kumada, H., Horiguchi, Y., 2000. The BNCT irradiation facility of JRR-4, Abstracts of ninth international symposium on neutron capture therapy for cancer, 242.

Yamamoto, T., Nakai, K., Kageji, T., Kumada, H., Endo, K., Matsuda, M., Shibata, Y., Matsumura, A., 2009. Boron neutron capture therapy for newly diagnosed glioblastoma. Radiother. Oncol. 91 (1), 80-84.

Yanagi e, H., Kumada, H., Sakurai, Y., Nakamura, T., Furuya, Y., Sugiyama, H., Ono, K., Takamoto, S., Eriguchi, M., Takahashi, H., 2009. Dosimetric evaluation of neutron capture therapy for local advanced breast cancer. Appl. Radiat. Isot. 67 (7-8), S63-S66.

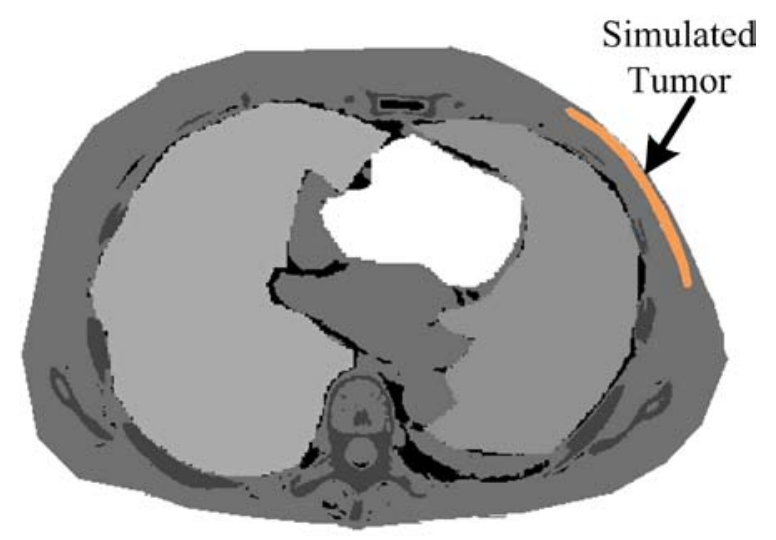

Figure 1 Cross section of evaluation model for the recurrent breast cancer. 
(a) En face technique
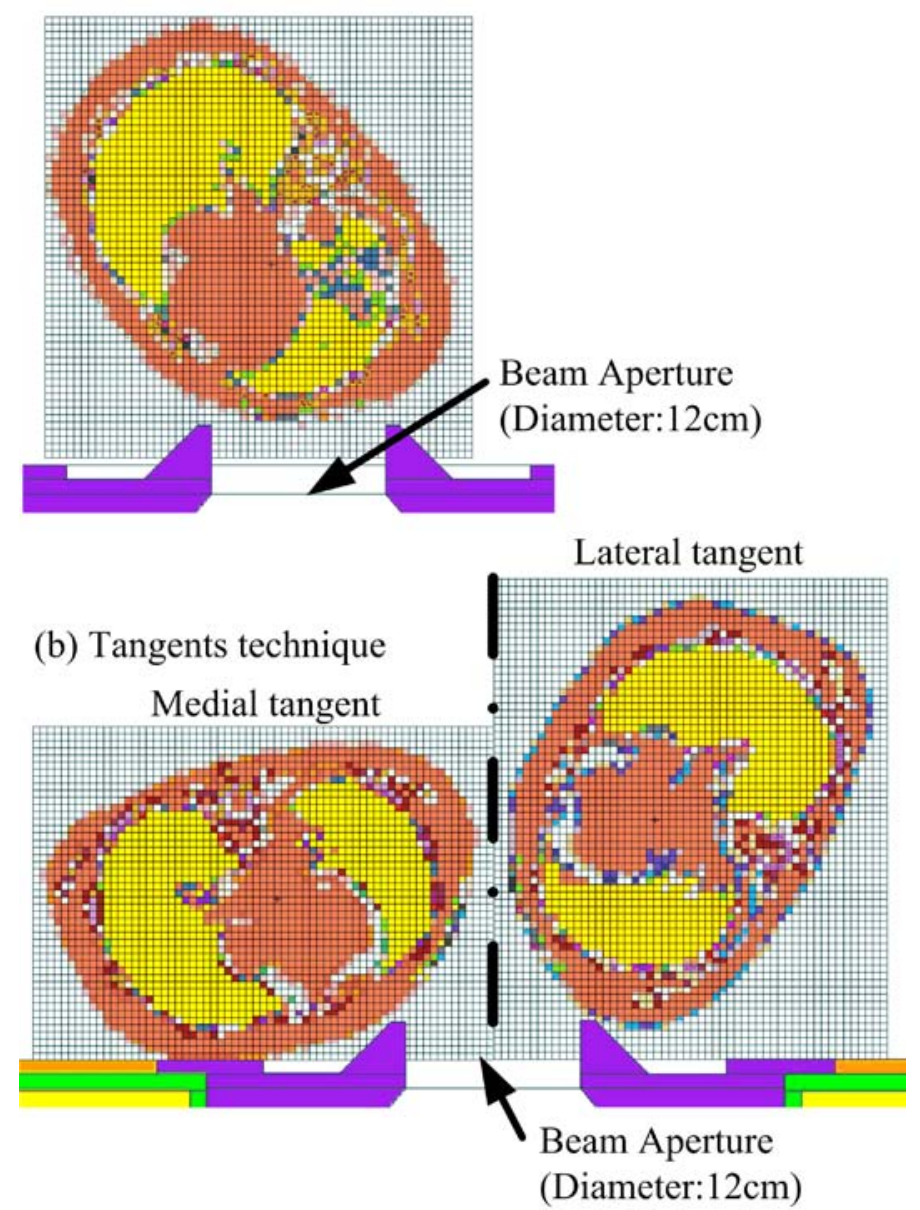

Figure 2 Irradiation setting model. (a) En face technique, (b) Tangents technique.

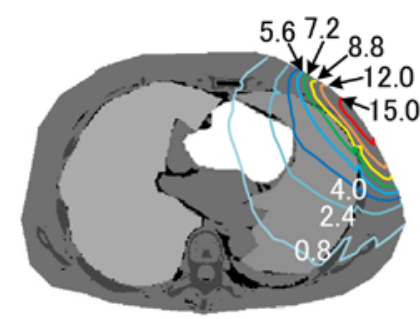

(a) En face technique

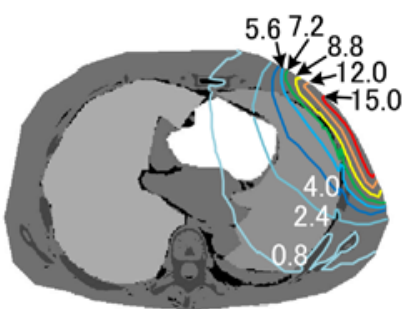

(b) Tangents technique

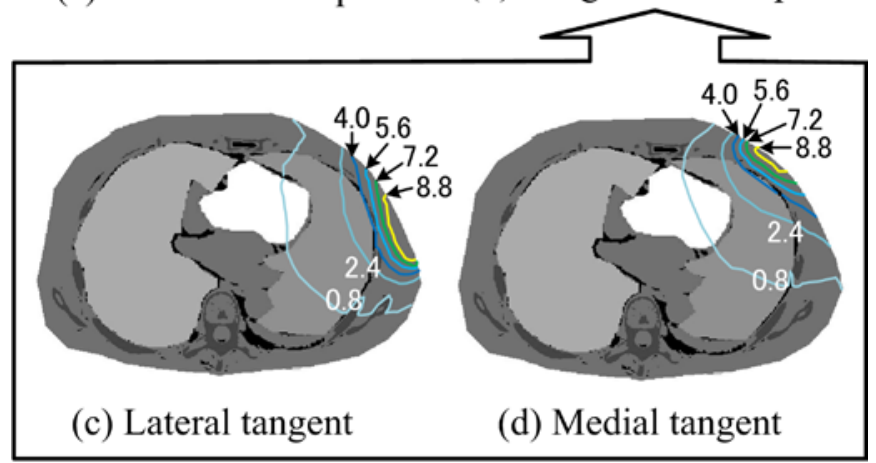

unit: Gy-Eq

Figure 3 Comparison of isodose distributions of lung. (a) En face technique, (b) Tangents technique, (c) Lateral tangent field, (d) Medial tangent field. 
Table 1 CBE factor, RBE factors and boron concentrations for this evaluation.

\begin{tabular}{|c|c|c|c|c|c|c|}
\hline & & Tumor & Skin & Lung & Liver & Heart \\
\hline \multicolumn{2}{|c|}{$\begin{array}{c}\text { Boron } \\
\text { concentration } \\
(\mu \mathrm{g} / \mathrm{g})\end{array}$} & 72 & 28.8 & 24 & 24 & 24 \\
\hline $\mathrm{CBE}$ & $\begin{array}{c}\text { Boron } \\
\text { dose }\end{array}$ & 3.8 & 2.5 & 2.3 & 4.25 & 1.35 \\
\hline \multirow{3}{*}{$\mathrm{RBE}$} & $\begin{array}{c}\text { Fast } \\
\text { neutron } \\
\text { dose }\end{array}$ & & & 2.5 & & \\
\hline & $\begin{array}{c}\text { Nitrogen } \\
\text { dose }\end{array}$ & & & 2.5 & & \\
\hline & $\begin{array}{c}\text { Gamma } \\
\text { dose }\end{array}$ & & & 1.0 & & \\
\hline
\end{tabular}

Table 2 Equivalent dose for the tumor and healthy organs and irradiation time.

\begin{tabular}{|c|c|c|c|}
\hline \multicolumn{2}{|c|}{ Irradiation technique } & En face & Tangents \\
\hline \multicolumn{2}{|c|}{ Irradiation time } & $40.2 \mathrm{~min}$ & $118.7 \mathrm{~min}$ \\
\hline \multirow{3}{*}{$\begin{array}{c}\text { Tumor Dose } \\
\text { (Gy-Eq) }\end{array}$} & $\mathrm{D}_{\max }$ & 65.0 & 66.8 \\
\hline & $\mathrm{D}_{\text {mean }}$ & 54.6 & 50.1 \\
\hline & $\mathrm{D}_{\min }$ & 30.0 & 30.0 \\
\hline $\begin{array}{l}\text { Skin Dose } \\
\text { (Gy-Eq) }\end{array}$ & $\mathrm{D}_{\max }$ & 18.2 & 19.5 \\
\hline \multirow{3}{*}{$\begin{array}{c}\text { Left Lung } \\
\text { Dose } \\
\text { (Gy-Eq) }\end{array}$} & $\mathrm{D}_{\max }$ & 9.8 & 8.3 \\
\hline & $\mathrm{D}_{\text {mean }}$ & 1.8 & 1.8 \\
\hline & $\mathrm{D}_{\min }$ & 0.2 & 0.3 \\
\hline \multirow{3}{*}{$\begin{array}{l}\text { Heart Dose } \\
\text { (Gy-Eq) }\end{array}$} & $\mathrm{D}_{\max }$ & 3.7 & 3.4 \\
\hline & $\mathrm{D}_{\text {mean }}$ & 0.7 & 0.7 \\
\hline & $\mathrm{D}_{\min }$ & 0.1 & 0.2 \\
\hline \multirow{3}{*}{$\begin{array}{l}\text { Liver Dose } \\
\text { (Gy-Eq) }\end{array}$} & $\mathrm{D}_{\max }$ & 2.0 & 2.1 \\
\hline & $\mathrm{D}_{\text {mean }}$ & 0.2 & 0.2 \\
\hline & $\mathrm{D}_{\min }$ & $<0.1$ & $<0.1$ \\
\hline
\end{tabular}

